LAPTH-701/98

hep-ph/9810273

\title{
THE HIGGS SECTOR OF THE MINIMAL 331 MODEL REVISITED
}

\author{
Nguyen Tuan Anh ${ }^{a}, \quad$ Nguyen Anh Ky ${ }^{b, c}$ and Hoang Ngoc Long ${ }^{b, d}$ 巴 \\ ${ }^{a}$ Graduate School, Institute of Physics, P. O. Box 429, Bo Ho, Hanoi 10000, Vietnam \\ ${ }^{b}$ Institute of Physics, NCNST, P. O. Box 429, Bo Ho, Hanoi 10000, Vietnam \\ ${ }^{c}$ LAPTH, Chemin de Bellevue, B.P. 110, F-74941 Annecy-le-Vieux, Cedex, France \\ ${ }^{d}$ Department of Physics, Chuo University, Kasuga, Bunkyo-ku, Tokyo 112-8551, Japan
}

\begin{abstract}
The mass spectrum and the eigenstates of the Higgs sector of the minimal 331 model are revisited in detail. There are discrepancies between our results and previous results by another author.
\end{abstract}

PACS numbers: 11.15.Ex, 12.60.Fr, 14.80.Cp.

\footnotetext{
${ }^{1}$ Fellow of the Japan Society for the Promotion of Science
} 


\section{Introduction}

At present, most high energy physicists recognize the great success of the Standard Model (SM) of electroweak and strong interactions. However, according to our present knowledge, the model is able to describe only the phenomenology at low energy below $200 \mathrm{GeV}$. In addition, one of the basic elements of the model is still an open problem, namely, the observation of the Higgs boson. Hence the mechanism for electroweak symmetry breaking is, in some way, still a mystery. The scalar sector plays an important role in many subjects of physics, and one of the most urgent problems in high energy physics is the search for the Higgs bosons. The scalar sector has been thoroughly studied not only in the SM framework but also in its various extensions. The models based on the $\mathrm{SU}(3)_{C} \otimes \mathrm{SU}(3)_{L} \otimes \mathrm{U}(1)_{N}(331)$ gauge group [1-6] is one of the most interesting extensions. These models have the following intriguing features: firstly, the models are anomaly free only if the number of families $\mathrm{N}$ is a multiple of three. Further, from the condition of QCD asymptotic freedom, which is valid only if the number of quark families is to be less than five, it follows that $\mathrm{N}$ is equal to 3. The second characteristic is that the Peccei-Quinn (PQ) [7] symmetry - a solution of the strong $\mathrm{CP}$ problem naturally occurs in these models [8]. It is worth mentioning that the implementation of the PQ symmetry is usually possible only at classical level (it will be broken by the quantum corrections through instanton effects), and there has been a number of attempts to find models for solving the strong CP question. In these models the PQ symmetry following from the gauge invariant Lagrangian does not have to be imposed. The third interesting feature is that one of the quark families is treated differently from the other two. This could lead to a natural explanation for the unbalancing heavy top quarks, deviations of $A_{b}$ from the SM prediction etc. In addition, the models predict no very high new mass scales at the order of a few $\mathrm{TeV}$ [9].

The scalar sector of the minimal 331 model was studied recently by Tonasse [10]. Three Higgs triplets were firstly analysed, then the sextet was added in a further consideration. Unfortunately, the last case - the three triplet and one sextet was presented so briefly such that the reader could hardly understand how it was obtained. Comparing with our results, besides some minor mistakes (misprints, we guess) in [10], we also found several discrepancies in the multiplet structures and some of the conclusions, especially those from the graphic analysis. In the present paper, considering again the Higgs sector we correct and present the results in a systematic order so that they can be checked and used in further studies.

The Higgs potentials of the model, constraint equations and main notations are presented in section 2. Sec. 3 is devoted to solving the characteristic equations. Our results are summarised in the last section. The full expression of the potential with three triplets and one sextet is given in the Appendix A, while the results for the toy model - the three triplet model is presented in the Appendix B where we explain why our results differ from those obtained in [10]. 


\section{Higgs structure and potential}

In the original version of the 331 model [1] the Higgs sector consists of three triplets

$$
\eta=\left(\begin{array}{c}
\eta^{0} \\
\eta_{1}^{-} \\
\eta_{2}^{+}
\end{array}\right) \sim(1,3,0) ; \rho=\left(\begin{array}{c}
\rho^{+} \\
\rho^{0} \\
\rho^{++}
\end{array}\right) \sim(1,3,1) ; \quad \chi=\left(\begin{array}{c}
\chi^{-} \\
\chi^{--} \\
\chi^{0}
\end{array}\right) \sim(1,3,-1),
$$

where the numbers in the brackets denote the quantum numbers under $\mathrm{SU}(3)_{C}, \mathrm{SU}(3)_{L}$ and $\mathrm{U}(1)_{N}$ respectively.

The most general (i.e., renormalizable and gauge invariant) $\mathrm{SU}(3)_{L} \otimes \mathrm{U}(1)_{N}$ Higgs potential which we can write with the three triplets of the Eqs. (2.1) is given by

$$
\begin{aligned}
V_{T}(\eta, \rho, \chi) & =\mu_{1}^{2} \eta^{\dagger} \eta+\mu_{2}^{2} \rho^{\dagger} \rho+\mu_{3}^{2} \chi^{\dagger} \chi+\lambda_{1}\left(\eta^{\dagger} \eta\right)^{2}+\lambda_{2}\left(\rho^{\dagger} \rho\right)^{2}+\lambda_{3}\left(\chi^{\dagger} \chi\right)^{2} \\
& +\left(\eta^{\dagger} \eta\right)\left[\lambda_{4}\left(\rho^{\dagger} \rho\right)+\lambda_{5}\left(\chi^{\dagger} \chi\right)\right]+\lambda_{6}\left(\rho^{\dagger} \rho\right)\left(\chi^{\dagger} \chi\right)+\lambda_{7}\left(\rho^{\dagger} \eta\right)\left(\eta^{\dagger} \rho\right) \\
& +\lambda_{8}\left(\chi^{\dagger} \eta\right)\left(\eta^{\dagger} \chi\right)+\lambda_{9}\left(\rho^{\dagger} \chi\right)\left(\chi^{\dagger} \rho\right)+\left(\frac{f_{1}}{2} \varepsilon^{i j k} \eta_{i} \rho_{j} \chi_{k}+\text { h. c. }\right)
\end{aligned}
$$

where the $\mu$ 's, and $f_{1}$ are mass parameters and coupling constants having a dimension of mass, while $\lambda$ 's are dimensionless. The detailed analysis of this potential is given in Appendix $\mathrm{B}$ where we explain the reason why our results obtained here differ from those by Tonasse in [10].

It was found soon after that in this model not all of the leptons got a mass, and this problem was solved by adding to the scalar sector a sextet [2]

$$
S=\left(\begin{array}{ccc}
\sigma_{1}^{0} & s_{2}^{+} & s_{1}^{-} \\
s_{2}^{+} & s_{1}^{++} & \sigma_{2}^{0} \\
s_{1}^{-} & \sigma_{2}^{0} & s_{2}^{--}
\end{array}\right) \sim(1, \overline{6}, 0)
$$

To avoid unwanted terms which make the analysis of the Higgs sector more complicated and lead to nonzero Majorana neutrino masses, a discrete symmetry should be imposed (for details, see [2]). Thus, we have additional terms in the Higgs potential in Eq. (2.2). The new (modified) potential is

$$
\begin{aligned}
V_{S}(\eta, \rho, \chi, S) & =V_{T}+\mu_{4}^{2} \operatorname{Tr}\left(S^{\dagger} S\right)+\lambda_{10} \operatorname{Tr}^{2}\left(S^{\dagger} S\right)+\lambda_{11} \operatorname{Tr}\left[\left(S^{\dagger} S\right)^{2}\right] \\
& +\left[\lambda_{12}\left(\eta^{\dagger} \eta\right)+\lambda_{13}\left(\rho^{\dagger} \rho\right)+\lambda_{14}\left(\chi^{\dagger} \chi\right)\right] \operatorname{Tr}\left(S^{\dagger} S\right) \\
& +\frac{1}{2}\left(f_{2} \rho^{T} S \chi+\text { h.c. }\right),
\end{aligned}
$$

where $V_{T}$ is given in Eq. (2.2). Let the neutral scalars $\eta^{0}, \rho^{0}, \chi^{0}$, and $\sigma_{2}^{0}$ develop real vacuum expectation values (VEV's) $v, u, w$ and $v^{\prime}$, respectively. (The CP-phenomenology arising from complex vevs in the 331 models has been inverstigated in [11]). We rewrite the expansion of the scalar fields

$$
\eta^{0}=v+\xi_{\eta}+i \zeta_{\eta} ; \rho^{0}=u+\xi_{\rho}+i \zeta_{\rho} ; \chi^{0}=w+\xi_{\chi}+i \zeta_{\chi} ; \sigma_{2}^{0}=v^{\prime}+\xi_{\sigma}+i \zeta_{\sigma}
$$


As in [2] here we do not consider neutrino mass, hence $\sigma_{1}^{0}$ does not develop a vacuum expectation

$$
\sigma_{1}^{0}=\xi_{\sigma}^{\prime}+i \zeta_{\sigma}^{\prime}
$$

Following 12 we call a real part $\xi$ scalar and an imaginary one $\zeta$ - pseudoscalar. The pattern of the symmetry breaking is

$$
\mathrm{SU}(3)_{C} \otimes \mathrm{SU}(3)_{L} \otimes \mathrm{U}(1)_{N} \stackrel{\langle\chi\rangle}{\longmapsto} \mathrm{SU}(3)_{C} \otimes \mathrm{SU}(2)_{L} \otimes \mathrm{U}(1)_{Y} \stackrel{\langle\rho, \eta, S\rangle}{\longmapsto} \mathrm{SU}(3)_{C} \otimes \mathrm{U}(1)_{Q},
$$

and the VEV's are related to the standard model one $\left(v_{W}\right)$ as $v^{2}+u^{2}+v^{\prime 2}=v_{W}^{2}=(246$ Gev $)^{2}$.

At the first step of symmetry breaking, the large $\langle\chi\rangle$ will generate masses for exotic quarks and new heavy gauge bosons $Z^{\prime}, X^{ \pm \pm}, Y^{ \pm}$. The subsequent breaking of $\mathrm{SU}(2)_{L} \otimes \mathrm{U}(1)$ is accomplished with nonzero values of $\langle\rho\rangle$ and $\langle\eta\rangle$, such that $t, s$ and $d$ aquire masses proportional to the former, while $b, c$, and $u$ aquire masses proportional to the latter.

To keep the model consistent with low-energy phenomenology, the VEV $\langle\chi\rangle$ must be large enough. In this paper we will use the following assumption: VEV of the Higgs field at the first step of symmetry breaking is assumed to be much larger than those at the second step, i.e.,

$$
w \gg v, u, v^{\prime}
$$

For further use we write

$$
\begin{aligned}
\operatorname{Tr}\left(S^{\dagger} S\right) & =2\left(v^{\prime 2}+2 v^{\prime} \xi_{\sigma}+\xi_{\sigma}^{2}+\zeta_{\sigma}^{2}\right)+\xi_{\sigma}^{\prime 2}+\zeta_{\sigma}^{\prime 2}+2 s_{1}^{+} s_{1}^{-}+2 s_{2}^{+} s_{2}^{-}+s_{1}^{++} s_{1}^{--}+s_{2}^{++} s_{2}^{--}, \\
\operatorname{Tr}\left[\left(S^{\dagger} S\right)^{2}\right] & =\left(\xi_{\sigma}^{\prime 2}+\zeta_{\sigma}^{\prime 2}+s_{1}^{+} s_{1}^{-}+s_{2}^{+} s_{2}^{-}\right)^{2}+\left(v^{\prime 2}+2 v^{\prime} \xi_{\sigma}+\xi_{\sigma}^{2}+\zeta_{\sigma}^{2}+s_{2}^{+} s_{2}^{-}+s_{1}^{++} s_{1}^{--}\right)^{2} \\
& +\left(v^{\prime 2}+2 v^{\prime} \xi_{\sigma}+\xi_{\sigma}^{2}+\zeta_{\sigma}^{2}+s_{1}^{+} s_{1}^{-}+s_{2}^{++} s_{2}^{--}\right)^{2} \\
& +2\left[\left(v^{\prime 2}+2 v^{\prime} \xi_{\sigma}+\xi_{\sigma}^{2}+\zeta_{\sigma}^{2}\right) s_{1}^{+} s_{1}^{-}+\left(\xi_{\sigma}^{\prime 2}+\zeta_{\sigma}^{\prime 2}\right) s_{2}^{+} s_{2}^{-}+\sigma_{1}^{o} \sigma_{2}^{o} s_{1}^{+} s_{2}^{-}+\sigma_{1}^{o *} \sigma_{2}^{o *} s_{2}^{+} s_{1}^{-}\right. \\
& \left.+\sigma_{1}^{o *} s_{2}^{+} s_{2}^{+} s_{1}^{--}+\sigma_{1}^{o} s_{2}^{-} s_{2}^{-} s_{1}^{++}+\sigma_{2}^{o *} s_{1}^{-} s_{2}^{-} s_{1}^{++}+\sigma_{2}^{o} s_{1}^{+} s_{2}^{+} s_{1}^{--}+s_{2}^{+} s_{2}^{-} s_{1}^{++} s_{1}^{--}\right] \\
& +2\left[\left(v^{\prime 2}+2 v^{\prime} \xi_{\sigma}+\xi_{\sigma}^{2}+\zeta_{\sigma}^{2}\right) s_{2}^{+} s_{2}^{-}+\left(\xi_{\sigma}^{\prime 2}+\zeta_{\sigma}^{\prime 2}\right) s_{1}^{+} s_{1}^{-}+\sigma_{1}^{o} \sigma_{2}^{o} s_{1}^{+} s_{2}^{-}+\sigma_{1}^{o *} \sigma_{2}^{o *} s_{2}^{+} s_{1}^{-}\right. \\
& \left.+\sigma_{1}^{o^{*}} s_{1}^{-} s_{1}^{-} s_{2}^{++}+\sigma_{1}^{o} s_{1}^{+} s_{1}^{+} s_{2}^{--}+\sigma_{2}^{o *} s_{1}^{+} s_{2}^{+} s_{2}^{--}+\sigma_{2}^{o} s_{1}^{-} s_{2}^{-} s_{2}^{++}+s_{1}^{+} s_{1}^{-} s_{2}^{++} s_{2}^{--}\right] \\
& +2\left[\left(v^{\prime 2}+2 v^{\prime} \xi_{\sigma}+\xi_{\sigma}^{2}+\zeta_{\sigma}^{2}\right)\left(s_{1}^{++} s_{1}^{--}+s_{2}^{++} s_{2}^{--}\right)+\sigma_{2}^{o} \sigma_{2}^{o} s_{2}^{++} s_{1}^{--}+\sigma_{2}^{o *} \sigma_{2}^{o *} s_{1}^{++} s_{2}^{--}\right. \\
& \left.+\sigma_{2}^{o} s_{1}^{-} s_{2}^{-} s_{2}^{++}+\sigma_{2}^{o} s_{1}^{+} s_{2}^{+} s_{1}^{--}+\sigma_{2}^{o *} s_{1}^{+} s_{2}^{+} s_{2}^{--}+\sigma_{2}^{o *} s_{1}^{-} s_{2}^{-} s_{1}^{++}+s_{1}^{+} s_{1}^{-} s_{2}^{+} s_{2}^{-}\right], \quad(2.8)
\end{aligned}
$$

and the complete expression of $V_{S}$ is given in the Appendix A.

The requirement that in the shifted potential $V_{S}$, the linear terms in fields must be absent, gives us the following constraint equations in the tree level approximation

$$
\begin{array}{r}
\mu_{1}^{2}+2 \lambda_{1} v^{2}+\lambda_{4} u^{2}+\lambda_{5} w^{2}+2 \lambda_{12} v^{\prime 2}+\frac{f_{1} u w}{2 v}=0, \\
\mu_{2}^{2}+2 \lambda_{2} u^{2}+\lambda_{4} v^{2}+\lambda_{6} w^{2}+2 \lambda_{13} v^{\prime 2}+\frac{f_{1} v w}{2 u}+\frac{f_{2} v^{\prime} w}{2 u}=0,
\end{array}
$$




$$
\begin{gathered}
\mu_{3}^{2}+2 \lambda_{3} w^{2}+\lambda_{5} v^{2}+\lambda_{6} u^{2}+2 \lambda_{14} v^{\prime 2}+\frac{f_{1} v u}{2 w}+\frac{f_{2} u v^{\prime}}{2 w}=0, \\
\mu_{4}^{2}+2\left(2 \lambda_{10}+\lambda_{11}\right) v^{\prime 2}+\lambda_{12} v^{2}+\lambda_{13} u^{2}+\lambda_{14} w^{2}+\frac{f_{2} u w}{4 v^{\prime}}=0 .
\end{gathered}
$$

Substituting Eqs. (2.1), (2.3), (2.5) and (2.6) into (2.2) and (2.4), and diagonalizing, we will get a mass spectrum of Higgs bosons with mixings.

\section{Higgs eigenstates and mass spectrum}

Since $\sigma_{1}^{0}$ has not a VEV, the associated scalar $\xi_{\sigma}^{\prime}$ and pseudo-scalar $\zeta_{\sigma}^{\prime}$ do not mix with other fields (for details, see [12]) and we indeed have the physical field $9 H_{\sigma}^{\prime} \approx \xi_{\sigma}^{\prime}$ with mass

$$
m_{H_{\sigma}^{\prime}}^{2}=2 \lambda_{11} v^{\prime 2}+\frac{f_{2} u w}{4 v^{\prime}}
$$

In the $\xi_{\eta}, \xi_{\rho}, \xi_{\sigma}$ and $\xi_{\chi}$ basis the square mass matrix, after imposing the constraints (2.9), reads as

$$
M_{4 \xi}^{2}=\left(\begin{array}{cccc}
4 \lambda_{1} v^{2}-\frac{f_{1} u w}{2 v} & 2 \lambda_{4} v u+\frac{f_{1} w}{2} & 4 \lambda_{12} v v^{\prime} & 2 \lambda_{5} v w+\frac{f_{1} u}{2} \\
2 \lambda_{4} v u+\frac{f_{1} w}{2} & 4 \lambda_{2} u^{2}-\frac{w}{2 u}\left(f_{1} v+f_{2} v^{\prime}\right) & 4 \lambda_{13} u v^{\prime}+\frac{f_{2} w}{2} & 2 \lambda_{6} u w+\frac{f_{1} v}{2}+\frac{f_{2} v^{\prime}}{2} \\
4 \lambda_{12} v v^{\prime} & 4 \lambda_{13} u v^{\prime}+\frac{f_{2} w}{2} & -m_{\xi_{\sigma}}^{2} & 4 \lambda_{14} w v^{\prime}+\frac{f_{2} u}{2} \\
2 \lambda_{5} v w+\frac{f_{1} u}{2} & 2 \lambda_{6} u w+\frac{f_{1} v}{2}+\frac{f_{2} v^{\prime}}{2} & 4 \lambda_{14} w v^{\prime}+\frac{f_{2} u}{2} & -m_{\xi_{\chi}}^{2}
\end{array}\right),
$$

where $m_{\xi_{\sigma}}^{2} \equiv-8\left(2 \lambda_{10}+\lambda_{11}\right) v^{\prime 2}+\frac{f_{2} u w}{2 v^{\prime}}, m_{\xi_{\chi}}^{2} \equiv-4 \lambda_{3} w^{2}+\frac{u}{2 w}\left(f_{1} v-f_{2} v^{\prime}\right)$.

With this matrix, it is difficult to get a clear physical meaning. As in [10] a meaningful approximation is to impose $\left|f_{1}\right|,\left|f_{2}\right| \sim w$ and to maintain only terms of the second order in $w$ in Eq. (3.2) (This means that we are working in low-energy phenomenology). This procedure immediately gives us one physical field

$$
H_{\chi} \approx \xi_{\chi}
$$

with mass

$$
m_{H_{\chi}}^{2} \approx-4 \lambda_{3} w^{2}
$$

and the square mass matrix of $\xi_{\eta}, \xi_{\rho}, \xi_{\sigma}$ mixing

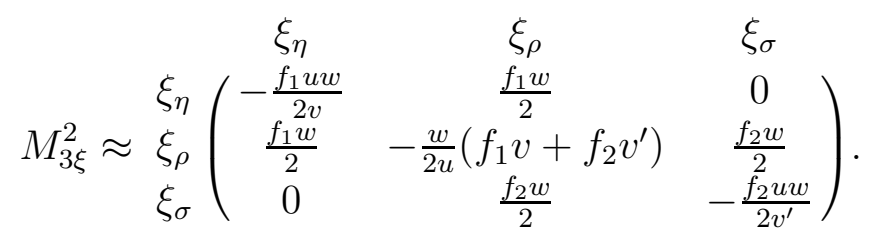

\footnotetext{
${ }^{2}$ Here we keep the notations in $[10]$ except the subscript 0 in eigenstates is omitted.
} 
Solving the charateristic equation of the matrix (3.5) we get one massless field $H_{1}$ and two physical ones $\left(\mathrm{H}_{2}, \mathrm{H}_{3}\right)$ with masses

$$
\begin{aligned}
x_{2,3} & =-\frac{w}{4}\left[\frac{f_{1}}{v u}\left(v^{2}+u^{2}\right)+\frac{f_{2}}{u v^{\prime}}\left(v^{\prime 2}+u^{2}\right)\right] \\
& \pm \frac{w}{4}\left\{\left[\frac{f_{1}}{v u}\left(v^{2}+u^{2}\right)+\frac{f_{2}}{u v^{\prime}}\left(v^{\prime 2}+u^{2}\right)\right]^{2}-\frac{4 f_{1} f_{2}}{v v^{\prime}} v_{W}^{2}\right\}^{1 / 2} \equiv m_{H_{2,3}}^{2} .
\end{aligned}
$$

Here, in Figs. 1 and 2, we plot $m_{02} \equiv m_{H_{2}} / w$ and $m_{03} \equiv m_{H_{3}} / w$ as functions of $u, v$ only

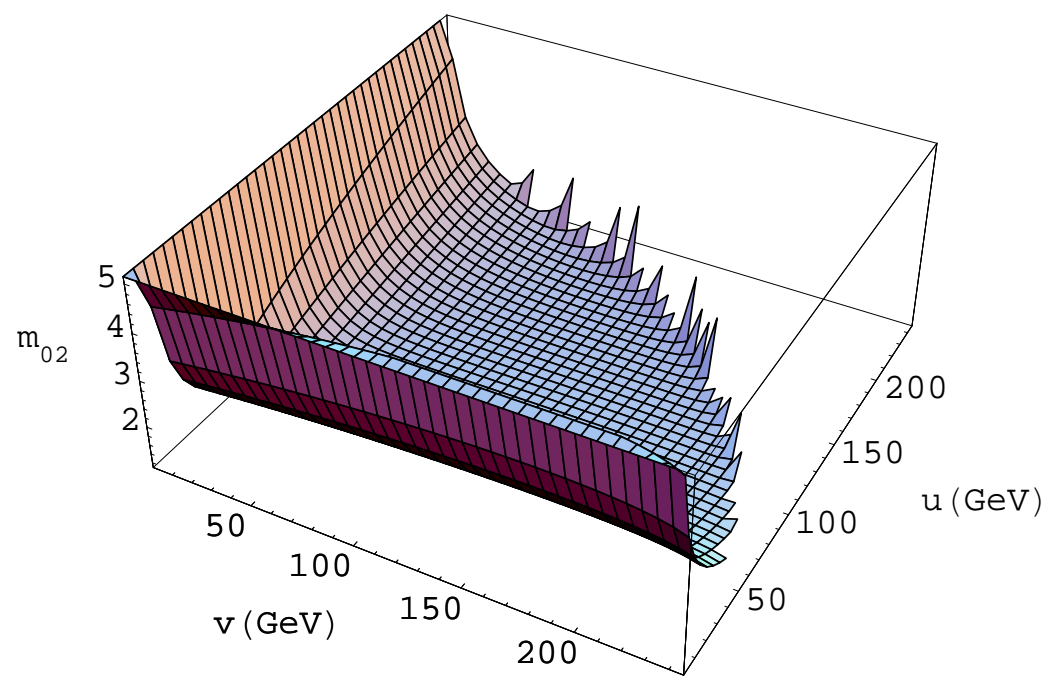

Figure 1: Behaviour of $m_{02}$ as a function of $u$ and $v$.

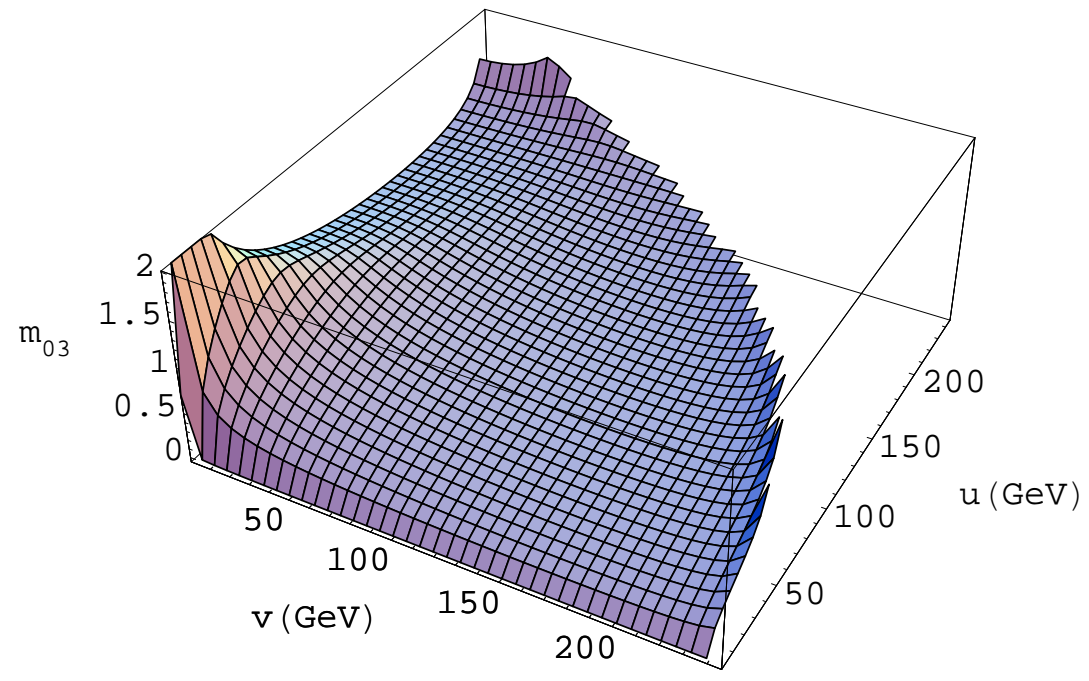

Figure 2: Behaviour of $m_{03}$ as a function of $u$ and $v$. 
since $v^{\prime}$ is given according to the constrain $u^{2}+v^{2}+v^{\prime 2}=246^{2}$. From the Figures, it follows that $u$ and $v$ are bounded as follows: $0<u, v \leq 240 \mathrm{GeV}$. Fig. 1 shows that $m_{H_{2}}$ increases when $u$ and $v$ tend to zero, while from Fig. 2 we see that $m_{H_{3}}$ vanishes at $u=0$ and increases when $v$ tends to zero and this mass is quite small. Note that our graphic surfaces are obtained in the suggestion $f_{1}, f_{2} \sim-w$ only. Otherwise, either $m_{03}$ is undefined (in the case of $f_{1}$ and $f_{2}$ having different signs) or both $m_{02}$ and $m_{03}$ are undefined (in case both $f_{1}$ and $f_{2}$ are positive).

The characteristic equation corresponding to $x_{2,3}$ can be written in the compact form

$$
u F_{1}(n) F_{2}(n)+v^{\prime} F_{1}(n)+v F_{2}(n)=0, \quad n=2,3,
$$

where

$$
F_{1}(i)=\frac{2 x_{i}}{f_{1} w}+\frac{u}{v} \text { and } F_{2}(i)=\frac{2 x_{i}}{f_{2} w}+\frac{u}{v^{\prime}}
$$

To construct physical fields (eigenstates) we begin from the charateristic equation

$$
\left(M^{2}-x_{i}\right) H_{i}=0, i=1,2,3,
$$

where $M^{2}$ is the considered square mass matrix and $H_{i} \equiv\left(H_{i 1}, H_{i 2}, H_{i 3}\right)^{T}$. With $M_{3 \xi}^{2}$ we have a system of three equations

$$
\begin{aligned}
-\left(\frac{f_{1} u w}{2 v}+x_{i}\right) H_{i 1}+\frac{f_{1} w}{2} H_{i 2} & =0, \\
\frac{f_{1} w}{2} H_{i 1}-\left[\frac{w}{2 u}\left(f_{1} v+f_{2} v^{\prime}\right)+x_{i}\right] H_{i 2}+\frac{f_{2} w}{2} H_{i 3} & =0, \\
\frac{f_{2} w}{2} H_{i 2}-\left(\frac{f_{2} u w}{2 v^{\prime}}+x_{i}\right) H_{i 3} & =0 .
\end{aligned}
$$

It is known [13 that this system of equations is over defined and must be reduced to two equations, in our case, the first and the last. Let us drop the second equation, and suppose

$$
H_{i 1}=k(i)
$$

where $k(i)$ will be defined by the normalization of the states [13.

From Eq. (3.10) we obtain

$$
H_{i 2}=\left(\frac{u}{v}+\frac{2 x_{i}}{f_{1} w}\right) k(i) \equiv F_{1}(i) k(i), \quad \text { (no summation over i). }
$$

Combining Eqs. (3.12) and (3.14) we then find

$$
H_{i 3}=\frac{F_{1}(i)}{F_{2}(i)} k(i)
$$


It is easy to see that from the condition of normalization of the states $H_{i}, \quad k(i)$ is found to be

$$
k(i)=\left[1+F_{1}(i)^{2}+\frac{F_{1}(i)^{2}}{F_{2}(i)^{2}}\right]^{-1 / 2} .
$$

Thus, we obtain finally a formula for the eigenstates of $\xi_{\eta}, \xi_{\rho}, \xi_{\sigma}$ mixing

$$
H_{i}=k(i)\left(\begin{array}{c}
1 \\
F_{1}(i) \\
F_{1}(i) / F_{2}(i)
\end{array}\right) \equiv\left(\begin{array}{c}
H_{i 1} \\
H_{i 2} \\
H_{i 3}
\end{array}\right)
$$

It is not difficult to verify that $H_{i}$ given in (3.17) are orthogonal to each other.

In the massless approximation $i=1$ (i.e., $x_{1}=0$ ) we immediately find

$$
H_{1}=\frac{1}{\sqrt{v^{2}+u^{2}+v^{\prime 2}}}\left(\begin{array}{c}
v \\
u \\
v^{\prime}
\end{array}\right) \text {. }
$$

In the next approximation (the $\lambda$ 's are taken into account) the field $H_{1}$ acquires a mass. Following [10] we solve the characteristic equation with the exact $3 \times 3$ mass matrix $M_{3 \xi}^{2}$, and the $H_{1}$ associated with, namely

$$
\left(M_{3 \xi}^{2}-x_{1}\right) H_{1}=0 .
$$

From system (3.19) we obtain the following formulas for the $H_{1}$ mass

$$
\begin{aligned}
m_{H_{1}}^{2} & =x_{1} \approx 4 \lambda_{1} v^{2}+2 \lambda_{4} u^{2}+4 \lambda_{12} v^{\prime 2} \approx 2 \lambda_{4} v^{2}+4 \lambda_{2} u^{2}+4 \lambda_{13} v^{\prime 2} \\
& \approx 4 \lambda_{12} v^{2}+4 \lambda_{13} u^{2}+8\left(2 \lambda_{10}+\lambda_{11}\right) v^{\prime 2} .
\end{aligned}
$$

From Eqs. (3.20) we can accept the following relation among coupling constants

$$
\lambda \approx \lambda_{1} \approx \lambda_{12} \approx \lambda_{4} / 2 \approx \lambda_{2} \approx \lambda_{13} \approx 2\left(2 \lambda_{10}+\lambda_{11}\right),
$$

and then the mass of $H_{1}$

$$
m_{H_{1}}^{2} \approx 4 \lambda\left(v^{2}+u^{2}+v^{\prime 2}\right) \equiv M_{01}^{2} .
$$

Thus, according to (3.3), (3.17) and (3.22), the eigenstates can be expressed as follows

$$
\begin{aligned}
\left(\begin{array}{c}
H_{1} \\
H_{2} \\
H_{3}
\end{array}\right) & \approx\left(\begin{array}{ccc}
2 \sqrt{|\lambda|} v / M_{01} & 2 \sqrt{|\lambda|} u / M_{01} & 2 \sqrt{|\lambda|} v^{\prime} / M_{01} \\
H_{21} & H_{22} & H_{23} \\
H_{31} & H_{32} & H_{33}
\end{array}\right)\left(\begin{array}{c}
\xi_{\eta} \\
\xi_{\rho} \\
\xi_{\sigma}
\end{array}\right), \\
H_{\chi} & \approx \xi_{\chi} .
\end{aligned}
$$

Since the matrix

$$
A_{H \xi}=\left(\begin{array}{ccc}
2 \sqrt{|\lambda|} v / M_{01} & 2 \sqrt{|\lambda|} u / M_{01} & 2 \sqrt{|\lambda|} v^{\prime} / M_{01} \\
H_{21} & H_{22} & H_{23} \\
H_{31} & H_{32} & H_{33}
\end{array}\right) \equiv\left(A_{H \xi}^{-1}\right)^{T}, \operatorname{det} A_{H \xi}=1
$$


in (3.23) is an orthogonal matrix $S O(3)$ the relation inverse to (3.23) and (3.24) can easily be found [14

$$
\begin{aligned}
\left(\begin{array}{c}
\xi_{\eta} \\
\xi_{\rho} \\
\xi_{\sigma}
\end{array}\right) & \approx\left(\begin{array}{ccc}
2 \sqrt{|\lambda|} v / M_{01} & H_{21} & H_{31} \\
2 \sqrt{|\lambda|} u / M_{01} & H_{22} & H_{32} \\
2 \sqrt{|\lambda|} v^{\prime} / M_{01} & H_{23} & H_{33}
\end{array}\right)\left(\begin{array}{c}
H_{1} \\
H_{2} \\
H_{3}
\end{array}\right), \\
\xi_{\chi} & \approx H_{\chi} .
\end{aligned}
$$

It is clear that our results here, especially the matrix in Eq. (3.26), are different from and more transparent than those given by Eqs. (19a)-(23b) in 10. In addition to this, the eigenstate corresponding to mass $-4 \lambda_{3} w^{2}$ is the scalar part of $\chi^{o}$, while in [10] it is the scalar part of $\sigma_{2}^{o}$.

Similarly, in the pseudoscalar sector we obtain one physical field $\zeta_{\sigma} \equiv \zeta_{\sigma}^{\prime}$ with a mass equal to the mass of $H_{\sigma}^{\prime}$, and the square mass matrix of the $\zeta_{\eta}, \zeta_{\rho}, \zeta_{\sigma}, \zeta_{\chi}$ mixing

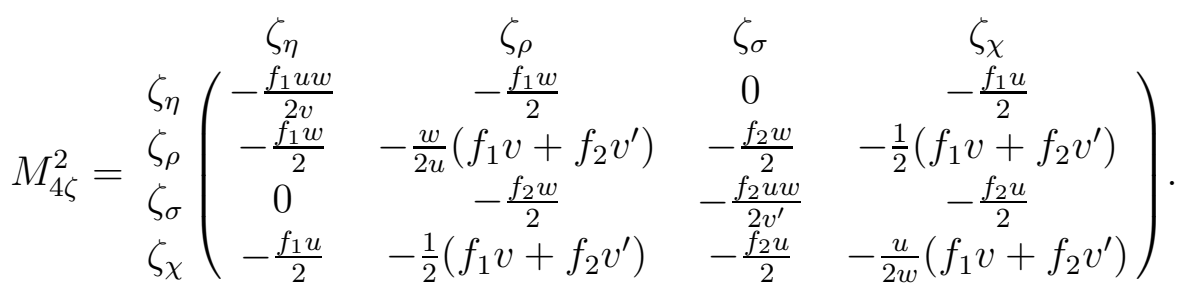

With the approximation as mentioned above we obtain one Goldstone boson $G_{1} \approx \zeta_{\chi}$ and the $\zeta_{\eta}, \zeta_{\rho}, \zeta_{\sigma}$ mixing

$$
M_{3 \zeta}^{2}=\zeta_{\eta} \zeta_{\rho}\left(\begin{array}{ccc}
\zeta_{\eta} & \zeta_{\rho} & \zeta_{\sigma} \\
\zeta_{\sigma} v & -\frac{f_{1} w}{2} & 0 \\
-\frac{f_{1} w}{2} & -\frac{w}{2 u}\left(f_{1} v+f_{2} v^{\prime}\right) & -\frac{f_{2} w}{2} \\
0 & -\frac{f_{2} w}{2} & -\frac{f_{2} u w}{2 v^{\prime}}
\end{array}\right) .
$$

It can be checked that the characteristic equation in this case have the same roots, but a different set of the eigenstates (simply, make a replace $H_{i 2} \rightarrow-H_{i 2}$ )

$$
\left(\begin{array}{c}
A_{1} \\
A_{2} \\
A_{3}
\end{array}\right) \approx\left(\begin{array}{ccc}
2 \sqrt{|\lambda|} v / M_{01} & -2 \sqrt{|\lambda|} u / M_{01} & 2 \sqrt{|\lambda|} v^{\prime} / M_{01} \\
H_{21} & -H_{22} & H_{23} \\
H_{31} & -H_{32} & H_{33}
\end{array}\right)\left(\begin{array}{c}
\zeta_{\eta} \\
\zeta_{\rho} \\
\zeta_{\sigma}
\end{array}\right)
$$

or equivalently

$$
\left(\begin{array}{c}
\zeta_{\eta} \\
\zeta_{\rho} \\
\zeta_{\sigma}
\end{array}\right) \approx\left(\begin{array}{ccc}
2 \sqrt{|\lambda|} v / M_{01} & H_{21} & H_{31} \\
-2 \sqrt{|\lambda|} u / M_{01} & -H_{22} & -H_{32} \\
2 \sqrt{|\lambda|} v^{\prime} / M_{01} & H_{23} & H_{33}
\end{array}\right)\left(\begin{array}{c}
A_{1} \\
A_{2} \\
A_{3}
\end{array}\right)
$$


In the singly charged sector the mixing occurs in the set of $\eta_{1}^{+}, \rho^{+}, s_{1}^{+}$and in the set of $\eta_{2}^{+}, \chi^{+}, s_{2}^{+}$(while in [10 the decompositions are $\eta_{1}^{+}, \rho^{+}, \eta_{2}^{+}$and $\chi^{+}, s_{1}^{+}, s_{2}^{+}$) with the following square mass matrices

$$
M_{+1}^{2}=\begin{gathered}
\eta_{1}^{-} \\
\rho^{-} \\
s_{1}^{-}
\end{gathered}\left(\begin{array}{ccc}
\lambda_{7} u^{2}-\frac{f_{1} u w}{2 v} & \rho_{7}^{+} v u-\frac{f_{1} w}{2} & s_{1}^{+} \\
\lambda_{7} v u-\frac{f_{1} w}{2} & \lambda_{7} v^{2}-\frac{w}{2 u}\left(f_{1} v+f_{2} v^{\prime}\right) & \frac{f_{2} w}{2} \\
0 & \frac{f_{2} w}{2} & -\frac{f_{2} u w}{2 v^{\prime}}
\end{array}\right),
$$

and

$$
M_{+2}^{2}=\begin{array}{ccc}
\eta_{2}^{+} & \chi^{+} & s_{2}^{+} \\
s_{2}^{-} & \chi^{-}
\end{array}\left(\begin{array}{ccc}
\lambda_{8} w^{2}-\frac{f_{1} u w}{2 v} & \lambda_{8} v w-\frac{f_{1} u}{2} & 0 \\
\lambda_{8} v w-\frac{f_{1} u}{2} & \lambda_{8} v^{2}-\frac{u}{2 w}\left(f_{1} v+f_{2} v^{\prime}\right) & \frac{f_{2} u}{2} \\
0 & \frac{f_{2} u}{2} & -\frac{f_{2} u w}{2 v^{\prime}}
\end{array}\right) .
$$

Applying the above approximation to $M_{+2}^{2}$ we obtain one Goldstone boson $G_{2}^{+} \approx \chi^{+}$and two physical fields associated with $\eta_{2}^{+}$and $s_{2}^{+}$and masses

$$
m_{\eta_{2}^{+}}^{2}=-\lambda_{8} w^{2}+\frac{f_{1} u w}{2 v}, m_{s_{2}^{+}}^{2}=\frac{f_{2} u w}{2 v^{\prime}} .
$$

For the $\eta_{1}^{+}, \rho^{+}, s_{1}^{+}$mixing, we have

$$
M_{+1}^{2}=\begin{array}{ccc}
\eta_{1}^{+} & \rho^{+} & s_{1}^{+} \\
\rho_{1}^{-} & s_{1}^{-}
\end{array}\left(\begin{array}{ccc}
-\frac{f_{1} u w}{2 v} & -\frac{f_{1} w}{2} & 0 \\
-\frac{f_{1} w}{2} & -\frac{w}{2 u}\left(f_{1} v+f_{2} v^{\prime}\right) & \frac{f_{2} w}{2} \\
0 & \frac{f_{2} w}{2} & -\frac{f_{2} u w}{2 v^{\prime}}
\end{array}\right) .
$$

As before, the characteristic equation of (3.35) has the same roots, but the eigenstates are different and are given by (necessary replaces: $H_{i 2} \rightarrow-H_{i 2}, H_{i 3} \rightarrow-H_{i 3}$ )

$$
\left(\begin{array}{c}
h_{1}^{+} \\
h_{2}^{+} \\
h_{3}^{+}
\end{array}\right) \approx\left(\begin{array}{ccc}
2 \sqrt{|\lambda|} v / M_{01} & -2 \sqrt{|\lambda|} u / M_{01} & -2 \sqrt{|\lambda|} v^{\prime} / M_{01} \\
H_{21} & -H_{22} & -H_{23} \\
H_{31} & -H_{32} & -H_{33}
\end{array}\right)\left(\begin{array}{c}
\eta^{+} \\
\rho^{+} \\
s_{1}^{+}
\end{array}\right)
$$

or equivalently

$$
\left(\begin{array}{c}
\eta^{+} \\
\rho^{+} \\
s_{1}^{+}
\end{array}\right) \approx\left(\begin{array}{ccc}
2 \sqrt{|\lambda|} v / M_{01} & H_{21} & H_{31} \\
-2 \sqrt{|\lambda|} u / M_{01} & -H_{22} & -H_{32} \\
-2 \sqrt{|\lambda|} v^{\prime} / M_{01} & -H_{23} & -H_{33}
\end{array}\right)\left(\begin{array}{c}
h_{1}^{+} \\
h_{2}^{+} \\
h_{3}^{+}
\end{array}\right)
$$

In the doubly charged sector the mixing occurs up all states $\rho^{++}, s_{2}^{++}, \chi^{++}, s_{1}^{++}$, and the square mass matrix is given

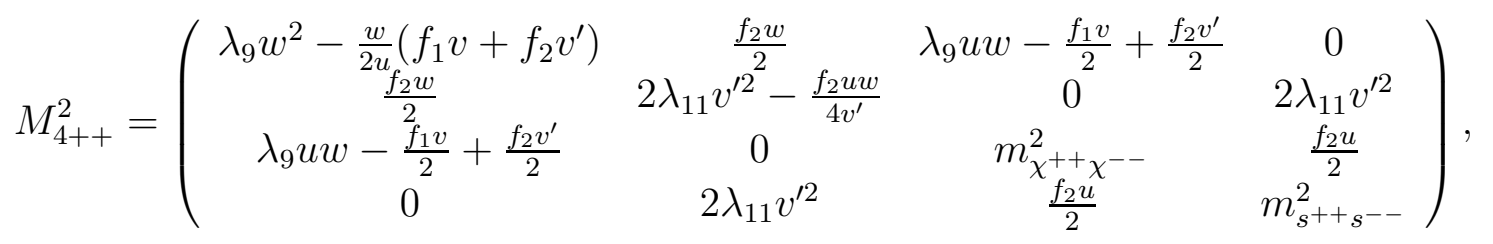


where $m_{\chi^{++} \chi^{--}}^{2} \equiv \lambda_{9} u^{2}-\frac{u}{2 w}\left(f_{1} v+f_{2} v^{\prime}\right)$ and $m_{s^{++} s^{--}}^{2} \equiv 2 \lambda_{11} v^{2}-\frac{f_{2} u w}{4 v^{\prime}}$.

By the same way as mentioned above we obtain one Goldstone boson $G_{3}^{++} \approx \chi^{++}$and one physical field $s_{1}^{++}$with mass

$$
m_{s_{1}^{++}}^{2}=\frac{f_{2} u w}{4 v^{\prime}}
$$

and a matrix of $\rho^{++}, s_{2}^{++}$mixing

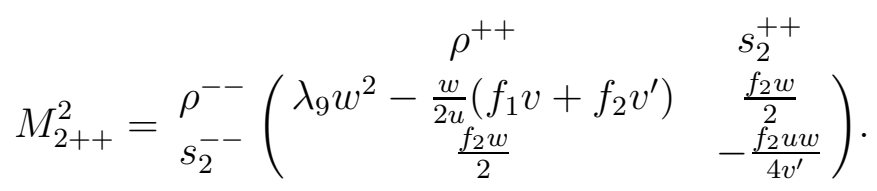

Solving the characteristic equation we get two physical fields

$$
\left(\begin{array}{c}
d_{1}^{++} \\
d_{2}^{++}
\end{array}\right)=\left(\begin{array}{cc}
n_{4}\left(\frac{u}{2 v^{\prime}}+\frac{2 x_{4}}{2 x_{2} w}\right), & n_{4} \\
n_{5}\left(\frac{u}{2 v^{\prime}}+\frac{2 x_{5}}{2 f_{2} w}\right), & n_{5}
\end{array}\right)\left(\begin{array}{c}
\rho++ \\
s_{2}^{++}
\end{array}\right),
$$

where

$$
n(i)=\left[1+\left(\frac{u}{2 v^{\prime}}+\frac{2 x_{i}}{f_{2} w}\right)^{2}\right]^{-\frac{1}{2}}(i=4,5),
$$

with masses

$$
\begin{aligned}
x_{4,5} & =\frac{w}{2}\left[\lambda_{9} w-\frac{f_{2}}{2}\left(\frac{u}{2 v^{\prime}}+\frac{v^{\prime}}{u}\right)-\frac{f_{1} v}{2 u}\right] \\
& \pm \frac{w}{2}\left\{\left[\lambda_{9} w-\frac{f_{2}}{2}\left(\frac{u}{2 v^{\prime}}+\frac{v^{\prime}}{u}\right)-\frac{f_{1} v}{2 u}\right]^{2}+\frac{\lambda_{9} f_{2} u w}{v^{\prime}}+\frac{f_{2}}{2 v^{\prime}}\left(f_{2} v^{\prime}-f_{1} v\right)\right\}^{1 / 2} .
\end{aligned}
$$

Let us summarize the particle content in the considered Higgs sector:

- in the neutral scalar sector physical fields are: $H_{1}, H_{2}, H_{3}, H_{\sigma}^{\prime}$ and $H_{\chi}$

$$
\begin{aligned}
& m_{H_{1}}^{2} \approx-4 \lambda v_{W}^{2}, m_{H_{2}}^{2}=x_{2}, m_{H_{3}}^{2}=x_{3}, \\
& m_{H_{\sigma}^{\prime}}^{2}=2 \lambda_{11} v^{2}+\frac{f_{2} u w}{4 v^{\prime}}, m_{\chi}^{2} \approx-4 \lambda_{3} w^{2},
\end{aligned}
$$

- in the neutral pseudoscalar sector, physical fields are: $A_{2}, A_{3}, A_{\sigma}$ and two Goldstone bosons: $G_{1} \approx \zeta_{\chi}$ and $G_{2}$ corresponding to the massless $A_{1}$

$$
m_{A_{2}}^{2}=x_{2}, m_{A_{3}}^{2}=x_{3}, m_{A_{\sigma}^{\prime}}^{2}=m_{H_{\sigma}^{\prime}}^{2},
$$

- in singly charged sector there are two Goldstone bosons $G_{3}=h_{1}^{+}, G_{4}^{+} \approx \chi^{+}$and three physical fields : $h_{2}^{+}, h_{3}^{+}, \eta_{2}^{+}, s_{2}^{+}$with masses:

$$
m_{h_{2}^{+}}^{2}=m_{H_{2}}^{2}, m_{h_{3}^{+}}^{2}=m_{H_{3}}^{2}, m_{\eta_{2}^{+}}^{2}=-\lambda_{8} w^{2}+\frac{f_{1} u w}{2 v}, m_{s_{2}^{+}}^{2}=\frac{f_{2} u w}{2 v^{\prime}},
$$


- in doubly charged sector we have one Goldstone $\left(G_{5}^{++} \approx \chi^{++}\right)$and three physical fields $d_{1}^{++}, d_{2}^{++}, s_{1}^{++}$with masses:

$$
m_{d_{1}^{++}}^{2}=x_{4}, m_{d_{2}^{++}}^{2}=x_{5}, m_{s_{1}^{++}}^{2}=\frac{f_{2} u w}{4 v^{\prime}} .
$$

Eqs. (3.44 - 3.47) show that $f_{2}$ has to be positive and there are three degenerate states $H_{2}, A_{2}$ and $h_{2}^{+}$in mass $x_{1}$, another three degenerate states $H_{3}, A_{3}$ and $h_{3}^{+}$in mass $x_{2}$, and two degenerate ones $H_{\sigma}^{\prime}, A_{\sigma}^{\prime}$ in mass $m_{H_{\sigma}^{\prime}}^{2}$. Again, eigenstates in these sectors (singly and doubly charged) are different from those in 10

Combining conditions for consistency (3.21) and positiveness of the mass square the following bounds for coupling constants are followed

$$
\begin{aligned}
\lambda \approx \lambda_{1} \approx \lambda_{12} \approx \lambda_{4} / 2 & \approx \lambda_{2} \approx \lambda_{13} \approx 2\left(2 \lambda_{10}+\lambda_{11}\right) \gtrsim 0, \\
\lambda_{3} & \lesssim 0 .
\end{aligned}
$$

It is worth mentioning here that the last relation $\lambda \approx 2\left(2 \lambda_{10}+\lambda_{11}\right)$ was replaced by $\lambda \approx$ $4\left(2 \lambda_{10}+\lambda_{11}\right)$ in [10] (see Eq. (21) there).

\section{Conclusion}

In conclusion, we have considered in this paper the Higgs sector of the minimal 331 model in the condition $\left|f_{1}\right|,\left|f_{2}\right| \sim w \gg v, u, v^{\prime}$. However, a consistent sign of $f_{2}$ is still under question: a positive $m_{H_{3}}^{2}$ needs negative $f_{1}$ and $f_{2}$ (see Eq. (3.6)), while expressions in Eqs. (3.34) - (3.47) require $f_{2}$ to be positive. This problem deserves further studies.

Other possibilities such as $f_{1}, f_{2} \sim v, u$ or $v^{\prime}$ cannot give us a simple solution. We do hope that further studies will justify this assumption.

It is to be mentioned that exposed in a more transparent way our results (eigenvalues and eigenstates) in the model of three triplets and one sextet, have some differences from those of the author of [10]. Our graphic surfaces give the conclusions, for example, $0<u, v \leq 240$ $\mathrm{GeV}$, quite different from those in [10]. However, there is a mass degeneracy in mass of scalar, pseudoscalar and singly charged Higgs fields with mass $m_{H_{3}}$ relatively small.

In the toy model - the three triplet model, the unnatural condition $v \neq u$ was removed. We hope that these differences will be examined in the future.

Note added: After submitting this paper for publication we have just been informed that some of our conclusions are in agreement with results in Ref. 11].

\section{Acknowledgments}

The authors thank D. Gomez Dumm for bringing their attention to Ref. [11] and for a discussion on some of the results. H. N. L. thanks T. Inami and Department of Physics, Chuo University for warm hospitality. N.A.K. thanks CNRS for financial support under the joint 
France-Vietnam Convention Internationale. He also thanks LAPTH for warm hospitality and P. Aurenche and G. Belanger for discussions.

This work was supported in part by the Research Programme on Natural Sciences of Hanoi National University under grant number QT 98.04 and KT - 04.1.1.

\section{Appendix A}

Here we give the full expression for the potential

$$
\begin{aligned}
& V_{S}(\eta, \rho, \chi, S)=\mu_{1}^{2}\left(v^{2}+2 v \xi_{\eta}+\xi_{\eta}^{2}+\zeta_{\eta}^{2}+\eta_{1}^{+} \eta_{1}^{-}+\eta_{2}^{+} \eta_{2}^{-}\right) \\
& +\mu_{2}^{2}\left(u^{2}+2 u \xi_{\rho}+\xi_{\rho}^{2}+\zeta_{\rho}^{2}+\rho^{+} \rho^{-}+\rho^{++} \rho^{--}\right) \\
& +\mu_{3}^{2}\left(w^{2}+2 w \xi_{\chi}+\xi_{\chi}^{2}+\zeta_{\chi}^{2}+\chi^{+} \chi^{-}+\chi^{++} \chi^{--}\right) \\
& +\lambda_{1}\left(v^{2}+2 v \xi_{\eta}+\xi_{\eta}^{2}+\zeta_{\eta}^{2}+\eta_{1}^{+} \eta_{1}^{-}+\eta_{2}^{+} \eta_{2}^{-}\right)^{2}+\lambda_{2}\left(u^{2}+2 u \xi_{\rho}+\xi_{\rho}^{2}+\zeta_{\rho}^{2}+\rho^{+} \rho^{-}+\rho^{++} \rho^{--}\right)^{2} \\
& +\lambda_{3}\left(w^{2}+2 w \xi_{\chi}+\xi_{\chi}^{2}+\zeta_{\chi}^{2}+\chi^{+} \chi^{-}+\chi^{++} \chi^{--}\right)^{2} \\
& +\left(v^{2}+2 v \xi_{\eta}+\xi_{\eta}^{2}+\zeta_{\eta}^{2}+\eta_{1}^{+} \eta_{1}^{-}+\eta_{2}^{+} \eta_{2}^{-}\right) \\
& \times\left[\lambda_{4}\left(u^{2}+2 u \xi_{\rho}+\xi_{\rho}^{2}+\zeta_{\rho}^{2}+\rho^{+} \rho^{-}+\rho^{++} \rho^{--}\right)\right. \\
& \left.+\lambda_{5}\left(w^{2}+2 w \xi_{\chi}+\xi_{\chi}^{2}+\zeta_{\chi}^{2}+\chi^{+} \chi^{-}+\chi^{++} \chi^{--}\right)\right] \\
& +\lambda_{6}\left(u^{2}+2 u \xi_{\rho}+\xi_{\rho}^{2}+\zeta_{\rho}^{2}+\rho^{+} \rho^{-}+\rho^{++} \rho^{--}\right)\left(w^{2}+2 w \xi_{\chi}+\xi_{\chi}^{2}+\zeta_{\chi}^{2}+\chi^{+} \chi^{-}+\chi^{++} \chi^{--}\right) \\
& +\lambda_{7}\left(\eta^{o 2} \rho^{+} \rho^{-}+\eta^{o} \rho^{o} \eta_{1}^{+} \rho^{-}+\eta^{o} \rho^{-} \eta_{2}^{-} \rho^{++}+\eta^{o *} \rho^{o *} \rho^{+} \eta_{1}^{-}+\rho^{o 2} \eta^{+} \eta^{-}\right. \\
& \left.+\rho^{o *} \eta_{1}^{-} \eta_{2}^{-} \rho^{++}+\eta^{o *} \rho^{+} \eta_{2}^{+} \rho^{--}+\rho^{o} \eta_{1}^{+} \eta_{2}^{+} \rho^{--}+\eta_{2}^{+} \eta_{2}^{-} \rho^{++} \rho^{--}\right) \\
& +\lambda_{8}\left(\eta^{o 2} \chi^{+} \chi^{-}+\chi^{o 2} \eta_{2}^{+} \eta_{2}^{-}+\eta^{o} \chi^{o} \eta_{2}^{-} \chi^{+}+\eta^{o *} \chi^{o *} \eta_{2}^{+} \chi^{-}+\eta^{o} \eta_{1}^{+} \chi^{+} \chi^{--}\right. \\
& \left.+\eta^{o *} \eta_{1}^{-} \chi^{-} \chi^{++}+\chi^{o *} \eta_{1}^{+} \eta_{2}^{+} \chi^{--}+\chi^{o} \eta_{1}^{-} \eta_{2}^{-} \chi^{++}+\eta_{1}^{+} \eta_{1}^{-} \chi^{++} \chi^{--}\right) \\
& +\lambda_{9}\left(\chi^{o 2} \rho^{++} \rho^{--}+\rho^{o 2} \chi^{++} \chi^{--}+\rho^{o} \chi^{o} \chi^{++} \rho^{--}+\rho^{o *} \chi^{o *} \rho^{++} \chi^{--}\right. \\
& \left.+\rho^{o} \rho^{-} \chi^{-} \chi^{++}+\rho^{o *} \rho^{+} \chi^{+} \chi^{--}+\chi^{o} \rho^{+} \chi^{+} \rho^{--}+\chi^{o *} \rho^{-} \chi^{-} \rho^{++}+\rho^{+} \rho^{-} \chi^{+} \chi^{-}\right) \\
& +\left\{\frac{f_{1}}{2}\left[\left(v+\xi_{\eta}+i \zeta_{\eta}\right)\left(\rho^{o} \chi^{o}-\rho^{++} \chi^{--}\right)-\eta_{1}^{-}\left(\chi^{o} \rho^{+}-\rho^{++} \chi^{--}\right)+\eta_{2}^{+}\left(\rho^{+} \chi^{--}-\rho^{o} \chi^{-}\right)\right]+\text {h.c }\right\} \\
& +\mu_{4}^{2}\left[2\left(v^{\prime 2}+2 v^{\prime} \xi_{\sigma}+\xi_{\sigma}^{2}+\zeta_{\sigma}^{2}\right)+\xi_{\sigma}^{\prime 2}+\zeta_{\sigma}^{\prime 2}+2 s_{1}^{+} s_{1}^{-}+2 s_{2}^{+} s_{2}^{-}+s_{1}^{++} s_{1}^{--}+s_{2}^{++} s_{2}^{--}\right] \\
& +\lambda_{10}\left[2\left(v^{\prime 2}+2 v^{\prime} \xi_{\sigma}+\xi_{\sigma}^{2}+\zeta_{\sigma}^{2}\right)+\xi_{\sigma}^{\prime 2}+\zeta_{\sigma}^{\prime 2}+2 s_{1}^{+} s_{1}^{-}+2 s_{2}^{+} s_{2}^{-}+s_{1}^{++} s_{1}^{--}+s_{2}^{++} s_{2}^{--}\right]^{2} \\
& +\left[2\left(v^{\prime 2}+2 v^{\prime} \xi_{\sigma}+\xi_{\sigma}^{2}+\zeta_{\sigma}^{2}\right)+\xi_{\sigma}^{\prime 2}+\zeta_{\sigma}^{\prime 2}+2 s_{1}^{+} s_{1}^{-}+2 s_{2}^{+} s_{2}^{-}+s_{1}^{++} s_{1}^{--}+s_{2}^{++} s_{2}^{--}\right] \\
& \times\left[\lambda_{12}\left(v^{2}+2 v \xi_{\eta}+\xi_{\eta}^{2}+\zeta_{\eta}^{2}+\eta_{1}^{+} \eta_{1}^{-}+\eta_{2}^{+} \eta_{2}^{-}\right)\right. \\
& +\lambda_{13}\left(u^{2}+2 u \xi_{\rho}+\xi_{\rho}^{2}+\zeta_{\rho}^{2}+\rho^{+} \rho^{-}+\rho^{++} \rho^{--}\right) \\
& \left.+\lambda_{14}\left(w^{2}+2 w \xi_{\chi}+\xi_{\chi}^{2}+\zeta_{\chi}^{2}+\chi^{+} \chi^{-}+\chi^{++} \chi^{--}\right)\right] \\
& +\left[\frac { f _ { 2 } } { 2 } \left(\chi^{-}\left(\sigma_{1}^{o} \rho^{+}+\rho^{o} s_{2}^{+}+\rho^{++} s_{1}^{-}\right)+\chi^{--}\left(\rho^{+} s_{2}^{+}+\rho^{o} s_{1}^{++}+\sigma_{2}^{o} \rho^{++}\right)\right.\right. \\
& \left.+\chi^{o}\left(\sigma_{2}^{o} \rho^{o}+\rho^{+} s_{1}^{-}+\rho^{++} s_{2}^{--}\right)+\text {h.c }\right]
\end{aligned}
$$




$$
\begin{aligned}
& +\lambda_{11}\left\{\left(\xi_{\sigma}^{\prime 2}+\zeta_{\sigma}^{\prime 2}+s_{1}^{+} s_{1}^{-}+s_{2}^{+} s_{2}^{-}\right)^{2}+\left(v^{\prime 2}+2 v^{\prime} \xi_{\sigma}+\xi_{\sigma}^{2}+\zeta_{\sigma}^{2}+s_{2}^{+} s_{2}^{-}+s_{1}^{++} s_{1}^{--}\right)^{2}\right. \\
& +\left(v^{\prime 2}+2 v^{\prime} \xi_{\sigma}+\xi_{\sigma}^{2}+\zeta_{\sigma}^{2}+s_{1}^{+} s_{1}^{-}+s_{2}^{++} s_{2}^{--}\right)^{2} \\
& +2\left[\left(v^{\prime 2}+2 v^{\prime} \xi_{\sigma}+\xi_{\sigma}^{2}+\zeta_{\sigma}^{2}\right) s_{1}^{+} s_{1}^{-}+\left(\xi_{\sigma}^{\prime 2}+\zeta_{\sigma}^{\prime 2}\right) s_{2}^{+} s_{2}^{-}+\sigma_{1}^{o} \sigma_{2}^{o} s_{1}^{+} s_{2}^{-}+\sigma_{1}^{o *} \sigma_{2}^{o *} s_{2}^{+} s_{1}^{-}\right. \\
& \left.+\sigma_{1}^{o *} s_{2}^{+} s_{2}^{+} s_{1}^{--}+\sigma_{1}^{o} s_{2}^{-} s_{2}^{-} s_{1}^{++}+\sigma_{2}^{o *} s_{1}^{-} s_{2}^{-} s_{1}^{++}+\sigma_{2}^{o} s_{1}^{+} s_{2}^{+} s_{1}^{--}+s_{2}^{+} s_{2}^{-} s_{1}^{++} s_{1}^{--}\right] \\
& +2\left[\left(v^{\prime 2}+2 v^{\prime} \xi_{\sigma}+\xi_{\sigma}^{2}+\zeta_{\sigma}^{2}\right) s_{2}^{+} s_{2}^{-}+\left(\xi_{\sigma}^{\prime 2}+\zeta_{\sigma}^{\prime 2}\right) s_{1}^{+} s_{1}^{-}+\sigma_{1}^{o} \sigma_{2}^{o} s_{1}^{+} s_{2}^{-}+\sigma_{1}^{o *} \sigma_{2}^{o *} s_{2}^{+} s_{1}^{-}\right. \\
& \left.+\sigma_{1}^{o^{*}} s_{1}^{-} s_{1}^{-} s_{2}^{++}+\sigma_{1}^{o} s_{1}^{+} s_{1}^{+} s_{2}^{--}+\sigma_{2}^{o *} s_{1}^{+} s_{2}^{+} s_{2}^{--}+\sigma_{2}^{o} s_{1}^{-} s_{2}^{-} s_{2}^{++}+s_{1}^{+} s_{1}^{-} s_{2}^{++} s_{2}^{--}\right] \\
& +2\left[\left(v^{\prime 2}+2 v^{\prime} \xi_{\sigma}+\xi_{\sigma}^{2}+\zeta_{\sigma}^{2}\right)\left(s_{1}^{++} s_{1}^{--}+s_{2}^{++} s_{2}^{--}\right)+\sigma_{2}^{o} \sigma_{2}^{o} s_{2}^{++} s_{1}^{--}+\sigma_{2}^{o *} \sigma_{2}^{o *} s_{1}^{++} s_{2}^{--}\right. \\
& \left.\left.+\sigma_{2}^{o} s_{1}^{-} s_{2}^{-} s_{2}^{++}+\sigma_{2}^{o} s_{1}^{+} s_{2}^{+} s_{1}^{--}+\sigma_{2}^{o *} s_{1}^{+} s_{2}^{+} s_{2}^{--}+\sigma_{2}^{o *} s_{1}^{-} s_{2}^{-} s_{1}^{++}+s_{1}^{+} s_{1}^{-} s_{2}^{+} s_{2}^{-}\right]\right\} .
\end{aligned}
$$

\section{Appendix B}

The constraint equations in the three triplet case (2.1) with the potential $V_{T}$ are

$$
\begin{aligned}
& \mu_{1}^{2}+2 \lambda_{1} v^{2}+\lambda_{4} u^{2}+\lambda_{5} w^{2}+\frac{f_{1} u w}{2 v}=0, \\
& \mu_{2}^{2}+2 \lambda_{2} u^{2}+\lambda_{4} v^{2}+\lambda_{6} w^{2}+\frac{f_{1} v w}{2 u}=0, \\
& \mu_{3}^{2}+2 \lambda_{3} w^{2}+\lambda_{5} v^{2}+\lambda_{6} u^{2}+\frac{f_{1} v u}{2 w}=0 .
\end{aligned}
$$

In the $\xi_{\eta}, \xi_{\rho}, \xi_{\chi}$ basis (which is ortho-normalized) the square mass matrix, after imposing the constraints (B.1), has the following form

$$
M_{\xi}^{2}=\xi_{\eta} \xi_{\rho}\left(\begin{array}{ccc}
8 \lambda_{1} v^{2}-f_{1} u w / v & \xi_{\rho} & \xi_{\chi} \\
4 \lambda_{4} v u+\lambda_{1} v u+f_{1} w & 8 \lambda_{5} v w+f_{1} u \\
4 \lambda_{5} v w+f_{1} u & 4 \lambda_{2} u^{2}-f_{1} v w / u+f_{1} v & 4 \lambda_{6} u w+f_{1} v \\
\xi_{\chi} & 8 \lambda_{3} w^{2}-f_{1} v u / w
\end{array}\right)\left(\frac{1}{2}\right) .
$$

Comparing with the results in [10] we notice that the mass matrix $M_{\xi}^{2}$ almost coincides with the mass matrix given by Eq. (4) in [10].

We will use the following approximation

$$
\left|f_{1}\right| \sim w, \quad w \gg v, u .
$$

Using (B.3) and keeping only the terms of second order in $w$, we got one massless $\left(H_{1}\right)$ and two massive physical states $\left(H_{2}\right.$ and $\left.H_{3}\right)$ with masses

$$
m_{H_{2}}^{2} \approx \frac{v^{2}+u^{2}}{2 v u} w^{2} \text { and } m_{H_{3}}^{2} \approx-4 \lambda_{3} w^{2}
$$

and mixing

$$
\left(\begin{array}{l}
\xi_{\eta} \\
\xi_{\rho}
\end{array}\right) \approx \frac{1}{\left(v^{2}+u^{2}\right)^{1 / 2}}\left(\begin{array}{cc}
v & -u \\
u & v
\end{array}\right)\left(\begin{array}{l}
H_{1} \\
H_{2}
\end{array}\right)
$$




$$
\xi_{\chi} \approx H_{3}
$$

In order to improve the approximation, following [10] we search the mass of $H_{1}$ by solving the characteristic equation with the exact $3 \times 3$ mass matrix $M_{\xi}^{2}$, and the $H_{1}$ associated with

$$
\left(M_{\xi}^{2}-m_{H_{1}}^{2}\right) H_{1}=0 .
$$

Solving a system of three equations (B.5) we get the mass for $H_{1}$

$$
m_{H_{1}}^{2} \approx 4 \frac{\lambda_{2} u^{4}-\lambda_{1} v^{4}}{v^{2}-u^{2}}
$$

and relations among the coupling constants and VEVs (if the approximation $f_{1} \approx-w$ is taken)

$$
\lambda_{4} \approx 2 \frac{\lambda_{2} u^{2}-\lambda_{1} v^{2}}{v^{2}-u^{2}}, \lambda_{5} v^{2}+\lambda_{6} u^{2} \approx-\frac{f_{1} v u}{2 w}=\frac{v u}{2} .
$$

Repeating the procedure for $H_{2}$ in a similar way we obtain an improved approximation for the mass $m_{\mathrm{H}_{2}}^{2}$

$$
m_{H_{2}}^{2} \approx 4 v^{2} u^{2} \frac{\lambda_{1}-\lambda_{2}}{v^{2}-u^{2}}+f_{1} w \frac{u^{2}+v^{2}}{u v}
$$

where the second term in (B.8) is the zero-approximation (B.4). Besides that we also obtain $\lambda_{4}$ given again as in (B.7) and

$$
\lambda_{5}-\lambda_{6} \approx f_{1} \frac{v^{2}-u^{2}}{4 v u w} \approx \frac{u^{2}-v^{2}}{4 v u} .
$$

We can solve (B.7) and (B.9) for $\lambda_{5}$ and $\lambda_{6}$

$$
\lambda_{5}=-\frac{f_{1} u}{4 v w} \approx \frac{u}{4 v}, \quad \lambda_{6}=-\frac{f_{1} v}{4 u w} \approx \frac{v}{4 u}, \quad \lambda_{5} \cdot \lambda_{6}=\left(\frac{f_{1}}{4 w}\right)^{2} \approx \frac{1}{16} .
$$

From (B.6) and (B.8) we see that if $v=u$ and $\lambda_{1} \neq \lambda_{2}$, the expressions of $m_{H_{1}}^{2}$ and $m_{H_{2}}^{2}$ become uncertain. It means that $v=u$ is a special case, and let us consider this one.

Setting $v=u$ and keeping only terms of second order in $w$, we got again one massless $\left(\hat{H}_{1}^{o}\right)$ and two massive physical states $\left(\hat{H}_{2}\right.$ and $\left.\hat{H}_{3}\right)$ with masses

$$
\begin{aligned}
& \hat{H}_{1} \approx \frac{1}{\sqrt{2}}\left(\xi_{\eta}+\xi_{\rho}\right) ; m_{\hat{H}_{1}}^{2}=0 \\
& \hat{H}_{2} \approx \frac{1}{\sqrt{2}}\left(-\xi_{\eta}+\xi_{\rho}\right) ; m_{\hat{H}_{2}}^{2}=w^{2}, \\
& \hat{H}_{3} \approx \xi_{\chi} ; m_{\hat{H}_{3}}^{2}=-4 \lambda_{3} w^{2} .
\end{aligned}
$$

From above equations, we see that eigenstates in this case are independent of $u$, and mass of $\hat{H}_{2}$ depend only on $w-\mathrm{VEV}$ of the heavy Higgs field $\chi$ at the first step of symmetry breaking. 
In order to improve the approximation, as before, we search mass of $\hat{H}_{1}$ by solving the following equation

$$
\left(\left.M_{\xi}^{2}\right|_{v=u}-m_{\hat{H}_{1}}^{2}\right) \hat{H}_{1}=0 .
$$

From Eq. (B.14) we get directly the following relations

$$
\begin{aligned}
\lambda_{1} & =\lambda_{2}, \\
m_{\hat{H}_{1}}^{2} & =2\left(2 \lambda_{1}+\lambda_{4}\right) u^{2}, \\
\lambda_{5}+\lambda_{6} & =-\frac{f_{1}}{2 w} \approx \frac{1}{2} .
\end{aligned}
$$

Now we consider the pseudoscalar sector. In the $\zeta_{\eta}, \zeta_{\rho}, \zeta_{\chi}$ basis (which is also orthonormalized) $M_{\zeta}^{2}$ takes the form

$$
M_{\zeta}^{2}=\zeta_{\rho}^{\zeta_{\eta}}\left(\begin{array}{ccc}
u / v & \zeta_{\rho} & \zeta_{\chi} \\
\zeta_{\chi} & v / u & v / w \\
u / w & v / w & v u / w^{2}
\end{array}\right)\left(-f_{1} w\right)
$$

It is easy to check that, the diagonalization of $M_{\zeta}^{2}$ gives us two Goldstone bosons $\left(G_{2}, G_{3}\right)$ and one massive pseudoscalar $A_{1}$ with exact mass

$$
m_{A_{1}}^{2}=-\frac{f_{1} w}{v u}\left[v^{2}+u^{2}+\left(\frac{v u}{w}\right)^{2}\right] .
$$

Let's denote the (normalized) eigenstate vectros $G_{1}, G_{2}$ and $G_{3}$ with their coordinates $X_{i}, Y_{i}, Z_{i}, \quad i=G_{1}, G_{2}, G_{3}$, as follows

$$
\left(\begin{array}{c}
X_{i} \\
Y_{i} \\
Z_{i}
\end{array}\right) \equiv X_{i} \zeta_{\eta}+Y_{i} \zeta_{\rho}+Z_{i} \zeta_{\chi}, \quad X_{i}^{2}+Y_{i}^{2}+Z_{i}^{2}=1
$$

The characteristic equations of $M_{\zeta}^{2}$ written in the forms

$$
\begin{gathered}
\frac{X_{n}}{v}+\frac{Y_{n}}{u}+\frac{Z_{n}}{w}=0, \quad n=G_{1}, G_{2} \\
v X_{G_{3}}=u Y_{G_{3}}=w Z_{G_{3}}
\end{gathered}
$$

show that the massless states $G_{1}$ and $G_{2}$ (orthogonal to each other, of course) belong to a plane orthogonal to the vector $(1 / v, 1 / u, 1 / w)^{T}$ which in turn is parallel to the massive state $G_{3}$

$$
G_{3}=\left(\begin{array}{c}
X_{G_{3}} \\
Y_{G_{3}} \\
Z_{G_{3}}
\end{array}\right)=N_{G_{3}}\left(\begin{array}{c}
1 / v \\
1 / u \\
1 / w
\end{array}\right)
$$


where

$$
N_{G_{3}}=\frac{u v w}{\left(v^{2} v^{2}+u^{2} w^{2}+v^{2} w^{2}\right)^{1 / 2}}
$$

is a normalization coefficient.

A plane can be parametrized by two parameters, say, $p$ and $q$. Putting (without losing generality)

$$
Y_{G_{1}}=p, \quad Z_{G_{1}}=q
$$

we get from $(\mathbb{B} .21)$

$$
G_{1}=\left(\begin{array}{c}
X_{G_{1}} \\
Y_{G_{1}} \\
Z_{G_{1}}
\end{array}\right)=N_{G_{1}}\left(\begin{array}{c}
-v[p / u+q / w] \\
p \\
q
\end{array}\right)
$$

where

$$
N_{G_{1}}=\frac{1}{\left[v^{2}(p / u+q / w)^{2}+p^{2}+q^{2}\right]^{1 / 2}}
$$

The state $G_{2}$ orthogonal to $G_{1}$ and $G_{3}$ can be determined (up to a sign) as the vector product

$$
G_{2}=G_{3} \times G_{1}
$$

written explicitly as

$$
G_{2}=\left(\begin{array}{c}
X_{G_{2}} \\
Y_{G_{2}} \\
Z_{G_{2}}
\end{array}\right)=N_{G_{2}}\left(\begin{array}{c}
q / u-p / w \\
-v[p / u+q / w] / w-q / v \\
p / v+v[p / u+q / w] / u
\end{array}\right)
$$

where

$$
N_{G_{2}}=N_{G_{1}} \cdot N_{G_{3}} .
$$

Note that the role of $G_{1}$ and $G_{2}$ can be exchanged.

Formulae $(\bar{B} .23),(\bar{B} .25)$ and $(\bar{B} .28)$ can be combined in a unique one as follows

$$
\left(\begin{array}{c}
G_{1} \\
G_{2} \\
G_{3}
\end{array}\right)=\left(\begin{array}{ccc}
X_{G_{1}} & Y_{G_{1}} & Z_{G_{1}} \\
X_{G_{2}} & Y_{G_{2}} & Z_{G_{2}} \\
X_{G_{3}} & Y_{G_{3}} & Z_{G_{3}}
\end{array}\right)\left(\begin{array}{c}
\zeta_{\eta} \\
\zeta_{\rho} \\
\zeta_{\chi}
\end{array}\right)
$$

where the matrix

$$
A=\left(\begin{array}{ccc}
X_{G_{1}} & Y_{G_{1}} & Z_{G_{1}} \\
X_{G_{2}} & Y_{G_{2}} & Z_{G_{2}} \\
X_{G_{3}} & Y_{G_{3}} & Z_{G_{3}}
\end{array}\right) \equiv\left(A^{-1}\right)^{T}, \quad \operatorname{det} A=1,
$$

is an orthogonal matrix $S O(3)$ and has the explicit form 


$$
A=\left(\begin{array}{ccc}
-v[p / u+q / w] N_{G_{1}} & p N_{G_{1}} & q N_{G_{1}} \\
{[q / u-p / w] N_{G_{2}}} & {[-v(p / u+q / w) / w-q / v] N_{G_{2}}} & {[v(p / u+q / w) / u+p / v] N_{G_{2}}} \\
N_{G_{3}} / v & N_{G_{3}} / u & N_{G_{3}} / w
\end{array}\right) .
$$

Then, the relation

$$
\left(\begin{array}{c}
\zeta_{\eta} \\
\zeta_{\rho} \\
\zeta_{\chi}
\end{array}\right)=\left(\begin{array}{ccc}
X_{G_{1}} & X_{G_{2}} & X_{G_{3}} \\
Y_{G_{1}} & Y_{G_{2}} & Y_{G_{3}} \\
Z_{G_{1}} & Z_{G_{2}} & Z_{G_{3}}
\end{array}\right)\left(\begin{array}{c}
G_{1} \\
G_{2} \\
G_{3}
\end{array}\right)
$$

is namely that inverse to (B.30).

As $w \gg u, v$, it is clear from Eq. (B.23) that there is no way to make the massive state $G_{3}$ parallel to the $\zeta_{\chi}-$ direction (i.e., to impose the condition $X_{G_{3}}=Y_{G_{3}}=0 \neq Z_{G_{3}}$ is impossible). It means that we always have $G_{3}$ different from $\zeta_{\chi}$ which could be singled out from $G_{1}$ and $G_{2}$ only. For example, in the limit $p \rightarrow 0$ and $w \rightarrow \infty \quad(w \gg u, v)$ we should have

$$
G_{1}=\left(\begin{array}{c}
0 \\
0 \\
1
\end{array}\right) \equiv \zeta_{\chi}, \quad G_{2}=\frac{1}{\left(u^{2}+v^{2}\right)^{1 / 2}}\left(\begin{array}{c}
v \\
-u \\
0
\end{array}\right), \quad G_{3}=\frac{1}{\left(u^{2}+v^{2}\right)^{1 / 2}}\left(\begin{array}{l}
u \\
v \\
0
\end{array}\right) .
$$

In contradiction to Tonasse's results, $\zeta_{\chi}$ here is not massive (as stated in [10]) but approximately massless, while $\zeta_{\eta}$ and $\zeta_{\rho}$ are mixings between the massless $G_{2}$ and the massive $G_{3}$ :

$$
\left(\begin{array}{l}
G_{2} \\
G_{3}
\end{array}\right)=\frac{1}{\left(v^{2}+u^{2}\right)^{1 / 2}}\left(\begin{array}{cc}
v & -u \\
u & v
\end{array}\right)\left(\begin{array}{l}
\zeta_{\eta} \\
\zeta_{\rho}
\end{array}\right), \quad G_{1}=\zeta_{\chi} .
$$

The inverse relation is

$$
\left(\begin{array}{l}
\zeta_{\eta} \\
\zeta_{\rho}
\end{array}\right)=\frac{1}{\left(v^{2}+u^{2}\right)^{1 / 2}}\left(\begin{array}{cc}
v & u \\
-u & v
\end{array}\right)\left(\begin{array}{c}
G_{2} \\
G_{3}
\end{array}\right), \quad \zeta_{\chi}=G_{1}
$$

The conclusion that $\zeta_{\chi}$ should be massless can be intuitively seen from the following observation: the mass terms associated with $\zeta_{\eta}, \zeta_{\rho}, \zeta_{\chi}$ fields in Higgs potential $V_{T}$, after imposing the constraints, are

$$
-\frac{f_{1} u w}{2 v},-\frac{f_{1} v w}{2 u},-\frac{f_{1} v u}{2 w}
$$

respectively. Therefore, in the limit $w \gg v, u$ the last term in (B.36) is smallest (massless).

The reason why our results differ from those of Tonasse is that Eqs. (10a) and (10b) in [10] do not represent relations between orthogonal bases as they should have to be. We 
would say the same for Eqs. (23a), (23b), (25a), (25b), (28a) and (28b) in that paper [10] when the sextet is included.

In the singly charged sector we have two Goldstone bosons and two physical massive fields with mixings

$$
\begin{aligned}
& \left(\begin{array}{c}
\eta_{1}^{+} \\
\rho^{+}
\end{array}\right)=\frac{1}{\left(v^{2}+u^{2}\right)^{1 / 2}}\left(\begin{array}{cc}
-v & u \\
u & v
\end{array}\right)\left(\begin{array}{c}
G_{4}^{+} \\
H_{5}^{+}
\end{array}\right), \\
& \left(\begin{array}{l}
\eta_{2}^{+} \\
\chi^{+}
\end{array}\right)=\frac{1}{\left(v^{2}+w^{2}\right)^{1 / 2}}\left(\begin{array}{cc}
-v & w \\
w & v
\end{array}\right)\left(\begin{array}{c}
G_{5}^{+} \\
H_{6}^{+}
\end{array}\right) .
\end{aligned}
$$

The masses of $H_{5}^{+}$and $H_{6}^{+}$are given, respectively

$$
m_{H_{5}^{+}}^{2}=\frac{v^{2}+u^{2}}{2 v u}\left(f_{1} w-2 \lambda_{7} v u\right), \quad m_{H_{6}^{+}}^{2}=\frac{u^{2}+w^{2}}{2 u w}\left(f_{1} u-2 \lambda_{8} u w\right) .
$$

Note, that at the considered (tree) level the mass spectrum and eigenstates in this sector are exact.

The doubly charged sector contains one Goldstone boson $G^{++}$and one physical massive scalar $H^{++}$

$$
\begin{aligned}
& \left(\begin{array}{c}
G^{++} \\
H^{++}
\end{array}\right)=\frac{1}{\left(u^{2}+w^{2}\right)^{1 / 2}}\left(\begin{array}{cc}
u & -w \\
w & u
\end{array}\right)\left(\begin{array}{l}
\rho^{++} \\
\chi^{++}
\end{array}\right), \\
& \left(\begin{array}{l}
\rho^{++} \\
\chi^{++}
\end{array}\right)=\frac{1}{\left(u^{2}+w^{2}\right)^{1 / 2}}\left(\begin{array}{cc}
u & w \\
-w & u
\end{array}\right)\left(\begin{array}{l}
G^{++} \\
H^{++}
\end{array}\right)
\end{aligned}
$$

with masses

$$
m_{G^{++}}=0, \quad m_{H^{++}}^{2}=\frac{u^{2}+w^{2}}{2 u w}\left(f_{1} v-2 \lambda_{9} u w\right)
$$

respectively.

Requiring that square masses of the physical fields are positive (otherwise, they are Goldstone ones) and combining Eqs.(B.4), (B.6), (B.8), (B.13), (B.39) and (B.42) we get the following relations between the parameters of the potential

$$
\begin{gathered}
\frac{\lambda_{1}}{\lambda_{2}}\left\{\begin{array}{l}
\lesssim u^{4} / v^{4}, \text { if } v>u, \\
\approx 1, \quad \text { if } v=u, \\
\gtrsim u^{4} / v^{4}, \text { if } v<u,
\end{array}\right. \\
\lambda_{3} \lesssim 0, f_{1}<0 ; \frac{f_{1}}{\lambda_{7}}<2 \frac{v u}{w}, \frac{f_{1}}{\lambda_{8}}<2 \frac{v w}{u}, \frac{f_{1}}{\lambda_{9}}<2 \frac{u w}{v} .
\end{gathered}
$$

Here the unnatural condition $v \neq u$ in $[10$ is removed. 


\section{References}

[1] F. Pisano and V. Pleitez, Phys. Rev. D46, 410 (1992);

P. H. Frampton, Phys. Rev. Lett. 69, 2889 (1992).

[2] R. Foot, O. F. Hernandez, F. Pisano, and V. Pleitez, Phys. Rev. D47, 4158 (1993).

[3] M. Singer, J. W. F. Valle, and J. Schechter, Phys. Rev. D22, 738 (1980).

[4] R. Foot, H. N. Long, and Tuan A. Tran, Phys. Rev. D50, R34 (1994).

[5] H. N. Long, Phys. Rev. D54, 4691 (1996).

[6] J. C. Montero, F. Pisano, and V. Pleitez, Phys. Rev. D47, 2918 (1993).

[7] R. D. Peccei and H. R. Quinn, Phys. Rev. Lett. 38, 1440 (1977); Phys. Rev. D16, 1791 (1977).

[8] P. B. Pal, Phys. Rev. D52, 1659 (1995).

[9] D. Ng, Phys. Rev. D49, 4805 (1994).

[10] M. D. Tonasse, Phys. Lett. B381, 191 (1996).

[11] D. G. Dumm, Int. J. Mod. Phys. A11, 887 (1996).

[12] H. N. Long, Mod. Phys. Lett. A13, 1865 (1998).

[13] V. I. Smirnov, A course of higher mathematics, vol. III, part one (Pergamon press, Oxford, 1964), pp $118-125$.

[14] G. A. Korn and T. M. Korn, Mathematical handbook for scientists and engineers, (McGraw-Hill Publishing Company, New York, 1968), pp 12 \& 407. 